\title{
1990-1991 Editorial consultants
}

The following is a list of editorial consultants who have reviewed original manuscripts for the JAOA or The DO during the past year. All are DOs, except as noted.

\section{A}

Robert C. Adams, Ft Worth, Tex Lee M. Adler, Orlando, Fla

Elliot L. Ames, Cherry Hill, NJ Robert D. Aranosian, Clarkston, Mich

\section{B}

Helen H. Baker, PhD, Chicago, IIl

Laurence H. Baker, Detroit, Mich

Edward P. Balaban, Dallas, Tex Donald A. Barone, Voorhees, NJ Robert I. Barsky, Stratford, NJ Myron C. Beal, Naples, NY John W. Becher, Jr, Philadelphia, $\mathrm{Pa}$

Laurence H. Belkoff, Wayne, Pa Frank Bellezza, PhD, Athens, Ohio

William E. Betts, Jr, Lancaster, $\mathrm{Pa}$

Gerald W. Blackburn, Farmington, Mich

Francis X. Blais, Ft Worth, Tex Gail D. Burchett, Kirksville, Mo

\section{C}

James Caffrey, PhD, Ft Worth, Tex

John Chiesa, Stratford, NJ

Anthony G. Chila, Athens, Ohio Michael B. Clearfield, Ft Worth, Tex

David Condoluci, Moorestown, NJ

Hugh H. Corddry, Warren, Mich James Cummings, Athens, Ohio

\section{D}

Gilbert E. D'Alonzo, Philadelphia, $\mathrm{Pa}$
Peter Dane, Athens, Ohio

Michael D. DeMattia, Mt Clemens, Mich

Stephen Dinsmore, Stratford, NJ

\section{E}

Joseph Eastman, Athens, Ohio Michael Emmett-Oglesby, PhD, Ft Worth, Tex

\section{F}

Harold P. Finkel, Bloomfield Hills, Mich

Arthur Freeman, PhD, Cherry Hills, NJ

Martha L. Friberg, Portland, Me Shelly A. Friedman, Scottsdale, $\mathrm{Az}$

Viola M. Frymann, LaJolla, Calif

Dean R. Fullingham, Tulsa, Okla

\section{G}

R. Michael Gallagher, Moorestown, NJ

Alan K. Geisler, Stratford, NJ

John P. Goodridge, East Lansing, Mich

Dennis Graham, Carney's Point, NJ

Robert Grant, Farmington Hills, Mich

Melvin Gray, PhD, MD, Chicago, Ill

George A. Gross, East Lansing, Mich

\section{H}

Dennis J. Hey, Kansas City, Mo Kari Hortos, Mt Clemens, Mich John N. Howell, PhD, Athens, Ohio

Charles G. Hughes, Colorado Springs, Colo

Frederick J. Humphrey, Stratford, NJ

\section{J}

Allen Jacobs, East Lansing, Mich

Sarah Jessup, Trenton, Mich
Joyce M. Johnson, Chevy Chase, Md

William L. Johnston, East Lansing, Mich

John Jones, Pomona, Calif

Donald A. Jurivich, LaGrange Park, Ill

\section{K}

Richard B. Kanoff, Wynnewood, $\mathrm{Pa}$

Neil M. Kantor, Dayton, Ohio Robert E. Kappler, Chicago, Ill Albert F. Kelso, PhD, Chicago, IIl

Donald Kirschner, Bala-Cynwyd, $\mathrm{Pa}$

George E. Kleiber, Lansing, Mich

Eugene Alex Kopple, Dayton, Ohio

Daniel Koprince, Royal Oak, Mich

David H. Krahe, Cleveland, Ohio

\section{L}

Sherman N. Leis, Bala-Cynwyd, $\mathrm{Pa}$

Michael T. C. Liang, PhD, Stratford, NJ

Ronald J. Librizzi, Philadelphia, $\mathrm{Pa}$

Robert D. Ligorsky, Phoenix, Az Richard F. Liszewski, Stratford, NJ

Robert Litchfield, Flossmoor, Ill Michael E. Lodish, Madison Heights, Mich

P. Robert Lombardo, Olympia Fields, Ill

Anne Loucks, $\mathrm{PhD}$, Athens, Ohio

\section{M}

Alex S. Macaione, Stratford, NJ Anthony J. Malcoun, Detroit, Mich

Ferdinand Manlio, Hollywood, Fla Mary Pat Mann, $\mathrm{PhD}$, Athens, Ohio

A. A. Mannarelli, Independence, Mo

Ronald V. Marino, Mineola, NY 


\section{0-1991 Editorial consultants \\ (continued)}

Timothy Martin, Monee, Ill Maj. Arthur S. Maslow, Tacoma, Wash

Michael McKenzie, Mt Clemens, Mich

Frederick G. Meoli, Cherry Hill, NJ Christopher Meyer, Flint, Mich Daniel Morrison, Garden City, Mich

Allan Morton, Warren, Mich

\section{N}

Alexander S. Nicholas, Philadelphia, $\mathrm{Pa}$

\section{O}

Michael I. Opipari, Mt Clemens, Mich

\section{$\mathbf{P}$}

Louis Papp, Olympia Fields, Ill Richard A. Pascucci, East Lansing, Mich

David A. Patriquin, Athens, Ohio

Michael M. Patterson, PhD, Athens, Ohio Andrew A. Pecora, Stratford, NJ Abraham Pera, Hemet, Calif Augustine L. Perrotta, Mt Clemens, Mich

George E. Piper, Jr, Haddonfield, NJ

Ronald Portanova, $\mathrm{PhD}$, Athens, Ohio
Lawrence L. Prokop, Washington, DC

\section{$\mathbf{R}$}

Richard Reilly, Tucson, Az

Louis E. Rentz, Farmington Hills, Mich

J. Jerry Rodos, Chicago, Ill

Felix J. Rogers, Trenton, Mich

Martin Roitman, Bridgeton, Mo

Rose Norman, Des Moines, Iowa

Randolphe G. Roulier, Toledo,

Ohio

Bernard Rubin, Ft Worth, Tex

\section{S}

Frederick Schaller, Stratford, NJ Donald J. Schnapf, Willow Street, $\mathrm{Pa}$

Harriet Shaw, Tulsa, Okla

Stephen H. Sheldon, Chicago, Ill Maj. William Shiels, Columbia, Md Lee R. Silverman, Southfield, Mich

Robert Simkins, Houston, Tex

Toni R. Smith, Kirksville, Mo

Stephen J. Sokalski, Oak Lawn, IIl

W. W. Stoever, Tulsa, Okla

Robert Stomel, Farmington Hills, Mich

Kenneth H. Stover, PhD, Los Osos, Calif

Stuart E. Strausberg, Panorama City, Calif
Kenneth Suarez, PhD, Downers Grove, Ill

Arthur Surloff, Hollywood, Fla

\section{$\mathbf{T}$}

Charles H. Tenner, Farmington Hills, Mich

Richard L. Theriault, Houston, Tex

\section{V}

William Vilensky, Cherry Hill, NJ

\section{W}

Matthew Weiss, Flint, Mich Paul E. Wenig, Troy, Mich Leonard A. Wilkerson, Kissimmee, Fla

Basil B. Williams, Jr, Ghent, NY David L. Wolf, Trenton, Mich

$\mathbf{Y}$

Gene Yonuschot, PhD, Biddeford, $\mathrm{Me}$

\section{Z}

T. Eugene Zachary, Ft Worth, Tex

Michael H. Zal, Bala-Cynwyd, $\mathrm{Pa}$

John J. Zuzga, Jr, Orland Park, Ill 